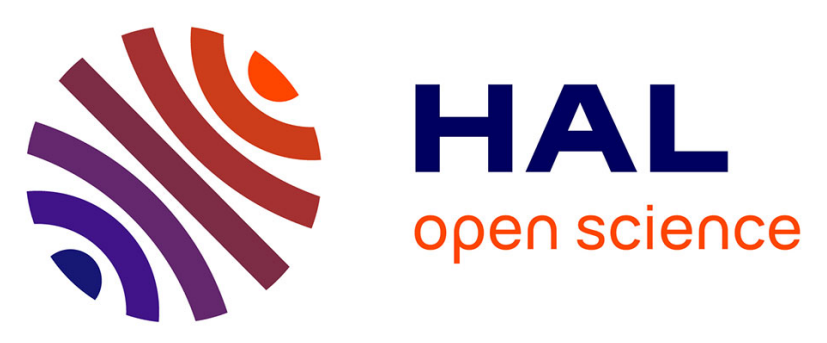

\title{
Segmentation-free and multiscale-free extraction of medial information using gradient vector flow - Application to vascular structures
}

Guillaume Pizaine, Raphaël Prevost, Elsa D. Angelini, Isabelle Bloch, Sherif Makram-Ebeid

\section{To cite this version:}

Guillaume Pizaine, Raphaël Prevost, Elsa D. Angelini, Isabelle Bloch, Sherif Makram-Ebeid. Segmentation-free and multiscale-free extraction of medial information using gradient vector flow - Application to vascular structures. Biomedical Imaging: Nano to Macro, 2012. 9th IEEE International Symposium on (ISBI 2012), May 2012, Barcelone, Spain. pp.780-783, 10.1109/ISBI.2012.6235533 . hal-00722958

\section{HAL Id: hal-00722958 \\ https://hal.science/hal-00722958}

Submitted on 6 Aug 2012

HAL is a multi-disciplinary open access archive for the deposit and dissemination of scientific research documents, whether they are published or not. The documents may come from teaching and research institutions in France or abroad, or from public or private research centers.
L'archive ouverte pluridisciplinaire HAL, est destinée au dépôt et à la diffusion de documents scientifiques de niveau recherche, publiés ou non, émanant des établissements d'enseignement et de recherche français ou étrangers, des laboratoires publics ou privés. 


\title{
SEGMENTATION-FREE AND MULTISCALE-FREE EXTRACTION OF MEDIAL INFORMATION USING GRADIENT VECTOR FLOW - APPLICATION TO VASCULAR STRUCTURES
}

\author{
Guillaume Pizaine $^{1,2}$ Raphael Prevost ${ }^{1,3} \quad$ Elsa D. Angelini ${ }^{2} \quad$ Isabelle Bloch $^{2} \quad$ Sherif Makram-Ebeid ${ }^{1}$ \\ ${ }^{1}$ Medisys Research Lab, Philips Healthcare, Suresnes, France. \\ ${ }^{2}$ Institut Telecom, Telecom ParisTech, CNRS LTCI, Paris, France. \\ ${ }^{3}$ CEREMADE, UMR 7534 CNRS, Paris Dauphine University, Paris, France.
}

\begin{abstract}
Gradient Vector Flow has become a popular method to recover medial information in medical imaging, in particular for vessels centerline extraction. This renewed interest has been motivated by its ability to proceed from gray-scale images, without prior segmentation. However, another interesting property lies in the diffusion process used to solve the corresponding variational problem. We propose a method to recover scale information in the context of vascular structures extraction, relying on analytical properties of the Gradient Vector Flow only, with no multiscale analysis. Through simple one-dimensional considerations, we demonstrate the ability of our approach to estimate the radii of the vessels with an error of $10 \%$ only in the presence of noise and less than $3 \%$ without noise. Our approach is evaluated on convolved bar-like templates and is illustrated on 2D X-ray angiographies.
\end{abstract}

Index Terms-gradient vector flow, diffusion, medialness, skeleton, shape analysis

\section{INTRODUCTION}

Gradient Vector Flow (GVF) has first been introduced as an external force field for active contours and active surfaces in $\mathrm{Xu}$ et al., 1998 [1]. The GVF of an image is the vector field obtained by diffusing image gradients in homogeneous regions while keeping strong gradients untouched. The diffusion process spreads edge information into uniform regions and acts as a long range force (see Fig. 1). Consequently, it also introduces more robustness against initialization and speeds up convergence.

Formally, the GVF of an image $I$ over a domain $\Omega$ is defined as the global minimizer $\boldsymbol{V}$ (Xu et al., 2000 [2]) of the following energy functional $E$ :

$$
E=\int_{\Omega} g(\boldsymbol{x})\|\nabla \boldsymbol{V}\|^{2}(\boldsymbol{x})+h(\boldsymbol{x})|\boldsymbol{V}(\boldsymbol{x})-\nabla I(\boldsymbol{x})|^{2} \mathrm{~d} \boldsymbol{x},
$$

where $g: \Omega \rightarrow \mathbb{R}$ and $h: \Omega \rightarrow \mathbb{R}$ are spatially-varying weighting functions and $\|\nabla \boldsymbol{V}\|$ is the vector norm for tensors given by $\sqrt{\nabla \boldsymbol{V} \cdot \nabla \boldsymbol{V}}$. The first term is a regularization term that controls the diffusion over the whole image domain. The second term is a data attachment term which ensures that $\boldsymbol{V}$ is close to the image gradient at strong edges. This is the General Gradient Vector Field (GGVF) devised by Xu et al., 1999 [3], which comes down to the original formulation of the GVF (Xu et al., 1998 [1]) if $g$ is constant and $h(\boldsymbol{x})=|\nabla I(\boldsymbol{x})|^{2}$. The most widely used functions are $g(\boldsymbol{x})=e^{-|\nabla I(\boldsymbol{x})|^{2} / K^{2}}, K \in \mathbb{R}$ and $h(\boldsymbol{x})=1-g(\boldsymbol{x})$, and will be used in this paper too. Since both formulations yield similar results, we will use the term GVF for both in the remaining of the paper.

The first variation of the functional $E$ yields the following EulerLagrange equation ${ }^{1}$ :

$$
g(\boldsymbol{x}) \Delta v_{i}(\boldsymbol{x})-h(\boldsymbol{x})\left(v_{i}(\boldsymbol{x})-\nabla I(\boldsymbol{x})\right)=0,
$$

where $v_{i}$ is the i-th component of the vector field and $\Delta$ is the Laplacian operator. The GVF is then the steady state of Eq. 2.

Recently, GVF has become popular in the field of medial information extraction. Many ways of using it have been proposed since it can be viewed as an improved gradient vector field to compute various features. For instance, Bauer et al., 2009 [4] propose to recover the centerlines of airways by computing the Hessian matrix from the GVF. Then, they determine the cross-sectional planes of the tubular structures and compute a tube-likeliness map from flux measures in those planes, based on the GVF, again. Flux measures were also used in Engel et al., 2008 [5] for medial features detection. Previous works also exhibit GVF-based medialness map derived from observations. Among them, the tube-likeliness from Bauer et al., 2009 [4] has already been mentioned. In Yu et al., 2004 [6], the authors propose to build a skeleton strength map from the GVF norm for grayscale image segmentation. Finally, the GVF has also been used to extract skeletons from binary shapes. In this context, the GVF is used in Hassouna et al., 2007 [7] in a front propagation setting to design a speed function allowing faster propagation at the center of structures.

Although the GVF has already been used to extract medial information, few works have proposed approaches to recover scale information. Unlike multiscale filters, which retain the maximum response over several scales, the GVF diffuses information without keeping track of the scale. Although one benefits from this by freeing oneself from scale constraints (e.g. Hessian matrices can be computed on a $3 \times 3$ neighborhood only), scale information is still paramount for skeletons or medialness maps. Knowing the centerlines, the method in Bauer et al., 2009 [4] goes back to the airways wall by tracking the GVF back to the edges in the image, which is quite time-consuming. In Engel et al., 2008 [5], the authors recover the size of the structures as the radius yielding a maximal circular (or spherical) flux. It seems in contradiction to the multiscale-free approach of the GVF.

In this paper, we propose a simple, segmentation-free and multiscale-free algorithm to extract medial information from images, based on the GVF. Since our approach heavily relies on

\footnotetext{
${ }^{1}$ As stated in Xu et al., 1999 [3], the calculus of variations yields a third term $\left\langle\nabla g(\boldsymbol{x}), \nabla v_{i}\right\rangle$ in the corresponding Euler-Lagrange equation, which does not change the result much in practice.
} 


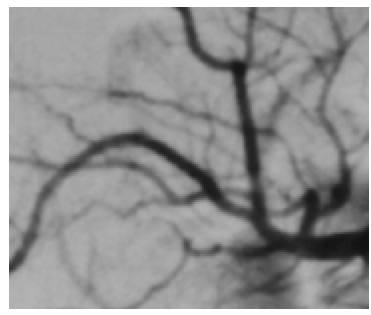

(a)

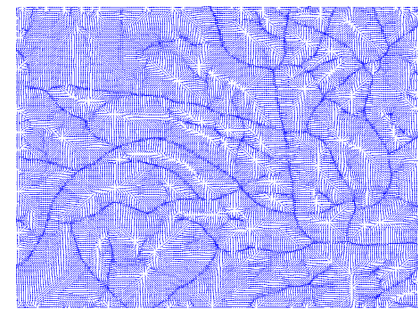

(b)
Fig. 1: Original image and its normalized GGVF.

one-dimensional analysis of the GVF (line by line in different directions), Sect. 2 gives a thorough review of the analytic solution to the one-dimensional case. Section 3 details the algorithm, especially how scale information is recovered. Finally, we discuss parameters and show results on 2D angiographies in Sect. 4.

\section{ANALYTICAL SOLUTION FOR THE ONE-DIMENSIONAL CASE}

Equation 2 is a diffusion-reaction equation whose analytical solution is not obvious without further assumptions on $h$ and $g$ (as defined in Sect. 1). For a better understanding, we will analyze the one-dimensional case. We consider edges as ramps which lead to plateau-like patterns in the original gradient (Fig. 2). The equation is decomposed and can be solved onto subdomains $\left\{\Omega_{k}\right\}_{0 \leq k \leq N}$ where $g_{k}$ and $h_{k}$, the restrictions of $g$ and $h$ to $\Omega_{k}$, are constant. In the following developments, $f_{k}$ will denote the restriction of a function $f$ to $\Omega_{k}$.

Two cases arise. If $\Omega_{k}$ is a homogeneous region, $\nabla I_{k}=0$ so $g_{k}(\boldsymbol{x})=1$ and $h_{k}(\boldsymbol{x})=0$. Equation 2 is then the one-dimensional heat equation $\frac{\partial^{2} V_{k}}{\partial x^{2}}=0$, so the solution is a linear function:

$$
V_{k}(x)=m_{k} x+p_{k}, \quad m_{k}, p_{k} \in \mathbb{R} .
$$

If $\Omega_{k}$ is a region where the gradient is non-zero, then $\nabla I_{k}$ is constant (due to the ramp model) and so are $g_{k}$ and $h_{k}$. Equation 2 has then the form

$$
\frac{\partial^{2} V_{k}}{\partial x^{2}}-a^{2}\left(V_{k}-\frac{\partial I}{\partial x}\right)=0, \quad a^{2}=\frac{1-g_{k}}{g_{k}}, \quad 0<g_{k} \leq 1
$$

Solutions to this second order linear equation with constant coefficients are of the form $V_{k}(x)=c_{k}^{(1)} e^{a x}+c_{k}^{(2)} e^{-a x}+b(x)$, where $c_{k}^{(1)}, c_{k}^{(2)} \in \mathbb{R}$ and $b$ is a particular solution. Since $\nabla I$ is constant over $\Omega_{k}$, it satisfies the equation. Finally, the solutions on such subdomains are of the form:

$$
V_{k}(x)=c_{k}^{(1)} e^{a x}+c_{k}^{(2)} e^{-a x}+\nabla I(x) .
$$

The parameters $m_{k}, p_{k}, c_{k}^{(1)}$ and $c_{k}^{(2)}$ for each subdomain $\Omega_{k}$ are given by the Dirichlet boundary condition $V=0$ on $\partial \Omega$, the $\mathcal{C}^{0}$ and the $\mathcal{C}^{1}$ properties of the global solution $V$ at boundaries between the $N$ subdomains. This yields the following linear system (in the same order):

$$
\begin{aligned}
p_{1} & =0 \\
m_{N} x_{N}+p_{N} & =0 \\
m_{k-1} x_{k}+p_{k-1} & =c_{k}^{(1)} e^{a x_{k}}+c_{k}^{(2)} e^{-a x_{k}}+v\left(x_{k}\right) \\
m_{k+1} x_{k+1}+p_{k+1} & =a c_{k}^{(1)} e^{a x_{k+1}}-a c_{k}^{(2)} e^{-a x_{k+1}}
\end{aligned}
$$

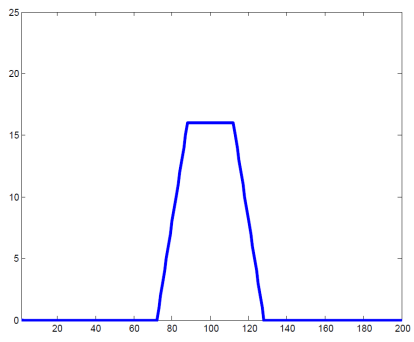

(a)

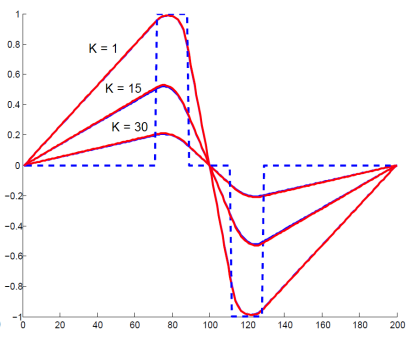

(b)
Fig. 2: (a) Original signal and (b) the analytical solution of the GVF equation for $K=3, K=15$ and $K=30$ (where $K$ is the parameter of function $g$ ). The dotted line represents the original normalized gradient, the analytical solution is plotted in plain red, and the numeric solution is in plain blue. Both solutions overlap almost completely. The zero-crossings are preserved for all values of $K$ but the positions of the maxima of the solution are clearly impacted.

where $x_{i}$ denotes the point limiting $\Omega_{i-1}$ and $\Omega_{i}$, and $0<k<N$. If there are $M$ plateau-like patterns, this yields a linear system of $4 M+2$ equations. A numerical solution and the corresponding analytical solution, computed from a two ramps gradient, are illustrated in Fig. 2. In practice, subdomains $\Omega_{k}$ where $\nabla I \neq 0$ tend towards $\varnothing$, which means that the GVF can be approximated by a piecewiselinear function. Although this is a mere approximation, we will use this property to derive our scale measure.

\section{DETECTION OF MEDIAL POINTS AND THEIR CORRESPONDING SCALE}

The GVF energy functional in Eq. 1 contains a diffusion term which is equivalent to a multiscale analysis, from a scale-space point of view. The method proposed here is driven by two ideas. First, scale information should be available directly from the GVF, without any further multiscale analysis. Second, since all the work has been done by the GVF, recovering scales should not use overcomplicated analysis schemes of the solution.

In contrast-enhanced images, vascular structures are considered as homogeneous regions surrounded by strong gradients. In those regions, the GVF matches gradients having opposite directions, in some sense. This interpretation still holds in the one-dimensional case: thanks to the separability property of the GVF, one can consider working on the projections of the solution $\boldsymbol{V}$ along each dimension instead of working on the gradient vector field itself. It means that analyzing the $d$-th component $V_{d}$ of $\boldsymbol{V}$ along the $d$-th dimension only is relevant. In this outlook, the separability of the GVF and results from Sect. 2 are exploited both to detect medial points and to estimate the radius of structures.

\subsection{Detection of medial points}

Matching gradients having opposite directions comes down to matching projections along each dimension $d$ having opposite signs (see Fig. 2). According to Sect. 2, the GVF may be approximated by a linear function and vanishes between those two gradients. To ensure that zero-crossings happen in the center of structures, both corresponding gradients must have exactly the same magnitude. This is why we choose to diffuse the normalized image gradient. In practice, the Point Spread Function (PSF) of the acquisition sys- 
tem interfere with the linearity of the solution inside homogeneous regions so that the slope of the solution $\boldsymbol{V}$ is weaker near edges. Along a given dimension $d$, medial points are thus detected as maxima of $\frac{\mathrm{d} V_{d}}{\mathrm{~d} x_{d}}$, which can still be emphasized by taking the normalized solution $\tilde{\boldsymbol{V}}$. Responses are summed over all dimensions to obtain the final measure for medial points:

$$
\mathcal{M}=\operatorname{div}(\tilde{\boldsymbol{V}})=\sum_{d} \frac{\mathrm{d} \tilde{V}_{d}}{\mathrm{~d} x_{d}} .
$$

\subsection{Estimation of the radius of the structures}

Following the remarks formulated in the previous paragraph concerning the linear approximation, the slope of $V_{d}$ is inversely proportional to the radius of the structures. Let $r_{d, k}$ be the size of the structures along dimension $d$, delimited by two matching gradients $V_{d}\left(x_{k}\right)$ and $V_{d}\left(x_{k+1}\right)$ at positions $x_{k}$ and $x_{k+1}$. The slope $m_{k}$ can be recovered where $V_{d}$ vanishes and the radius can be estimated as:

$$
r_{d, k}=\frac{V_{d}\left(x_{k}\right)-V_{d}\left(x_{k+1}\right)}{2 m_{k}} .
$$

Knowing the positions $x_{k}$ is not obvious. This is why previous works usually resort to an exhaustive search through multiscale analysis. On the contrary, since we are able to detect structures of interest thanks to zero-crossings, we have all the necessary information to approximate $V_{d}$ with a piecewise-linear function. We are only interested in the positions where two linear functions intersect, thus the approximation does not have to be accurate (see Fig. 3). A position $x_{k}$ responsible for linear regions $\Omega_{k}$ and $\Omega_{k+1}$ with corresponding zero-crossings $c_{k}$ and $c_{k+1}$ is thus recovered as:

$$
x_{k}=\frac{m_{k+1} c_{k+1}-m_{k} c_{k}}{m_{k+1}-m_{k}}
$$

The actual radius $r_{k}$ can now be computed with simple geometrical considerations. For example, for $2 \mathrm{D}$ images, the radius is:

$$
r_{k}=r_{1} \sin \arccos \left(\frac{r_{1}}{\sqrt{r_{1}^{2}+r_{2}^{2}}}\right) \text {, }
$$

where $r_{1}$ and $r_{2}$ are the radii estimated along each direction.

\section{EVALUATION OF THE ESTIMATED SCALES AND APPLICATION TO VASCULAR STRUCTURES}

Equation 2 can be solved with various explicit, implicit or semiimplicit schemes. We implemented the common explicit scheme for simplicity (see Boukerroui, 2009 [8] for more efficient explicit and implicit schemes). In particular, unconditionnally stable explicit schemes exist (the Alternating Direction Explicit scheme, for example). In practice, the straightforward explicit scheme is still widely used and is very useful for investigation. We recall this scheme (Xu et al., 1998 [3]):

$$
V_{i}^{n+1}=(1-h \Delta t) V_{i}^{n}+\frac{g \Delta t}{\Delta x}\left(V_{i-1}^{n}+V_{i+1}^{n}-2 V_{i}^{n}\right)+h \nabla I \Delta t,
$$

where $\Delta x$ is the spatial resolution.

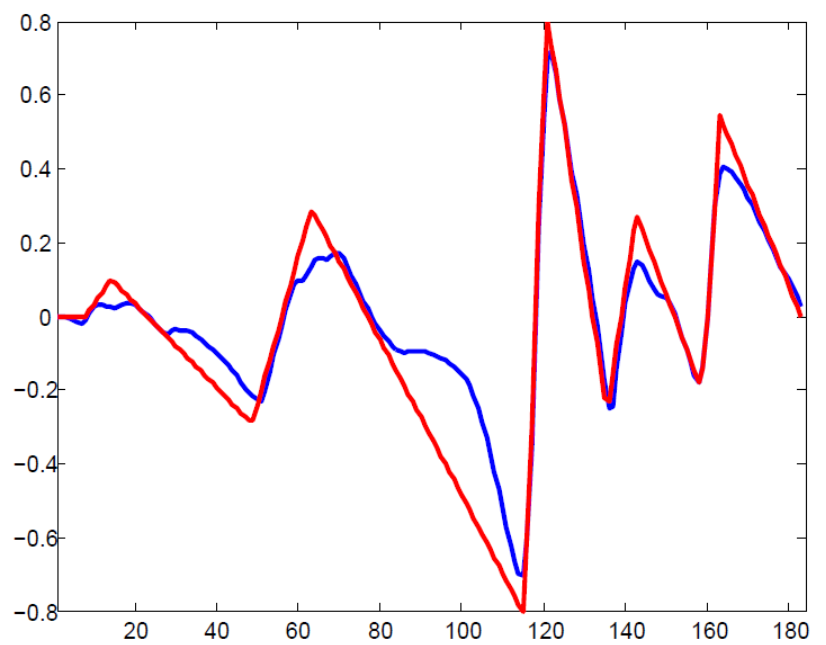

Fig. 3: Solution to the GVF (in blue) for a one-dimensional extracted from Fig. 1 and its corresponding piecewise linear reconstruction (in red).

\subsection{Validation on synthetical vessel templates}

As mentioned in Sect. 3.1, the PSF of the acquisition system and partial volume effects impact the estimation of the vessels radius. To study their influence, we apply our algorithm to vessel templates with various radii and PSF. Vessels are modeled by convolved barlike cross-sections with radii $r^{0}$ ranging from 1 to 25 pixels, and the scale of the convolution $\sigma_{P S F}$ is $0.5,1$ and 2 pixels (we approximate the PSF by a Gaussian distribution).

The relative error of the estimation with respect to the ground truth $\operatorname{err}(r)=\frac{\left|r-r^{0}\right|}{r^{0}}$ is illustrated in Fig. 4. The algorithm introduced in Sect. 3.2 is represented by blue lines. We compare it with two other approaches. The first one, represented by red lines, is the radius evaluated by taking $r=\min \left(\left|x_{k}-c_{k}\right|,\left|x_{k+1}-c_{k+1}\right|\right)$. The second one, represented by the green lines, correspond to the distance from $c_{k}$ to the closest local maximum of $\|\boldsymbol{V}\|$. Finally, the evaluation was performed on noise-free profiles $(K=5)$ in the first column, and on profiles with a $10 \%$ random additive Gaussian noise ( $K=15$ to compensate for the presence noise) in the second column.

It is clear that our algorithm performs better for all PSF values and is globally more robust to noise. When $r^{0} \leq \sigma_{P S F}$ with no noise, the estimation is clearly unreliable but usable since the error is still less than one pixel. For $r^{0}>\sigma_{P S F}$, the error is less than $3 \%$ for noise-free profiles, and remains low (around 10\%) in the presence of an additive Gaussian noise. However, for radii smaller than the PSF, zero-crossings of the GVF may disappear and thus our algorithm fails to recover the structure, which corresponds to the very high errors in Fig. 4.

\subsection{Skeleton extraction of vascular structures}

Our algorithm was also tested to extract the skeleton of vascular structures in 2D angiographies. The medialness map $\mathcal{M}$ from Eq.7 and the radii are computed from the $2 \mathrm{D} \mathrm{GVF}$ of the image. Seed points are selected as directional maxima of $\mathcal{M}$ and those lying in regions with low local contrast are discarded. Finally, centerlines are extracted as the ridges of $\mathcal{M}$ going through seed points. The 


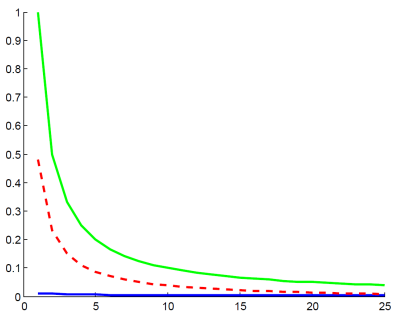

(a)

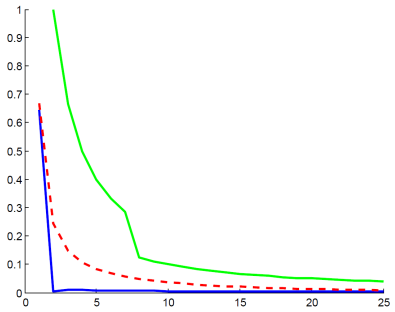

(c)

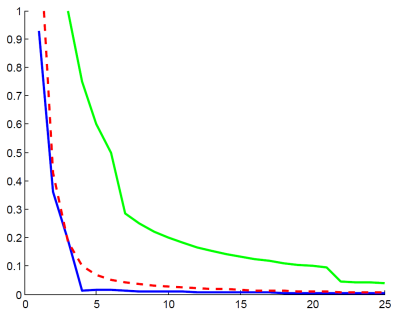

(e)

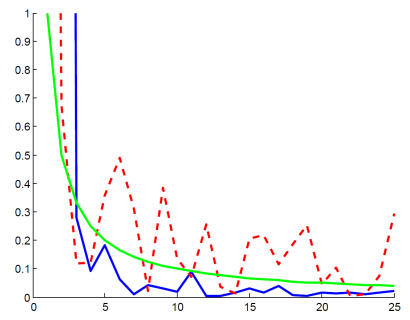

(b)

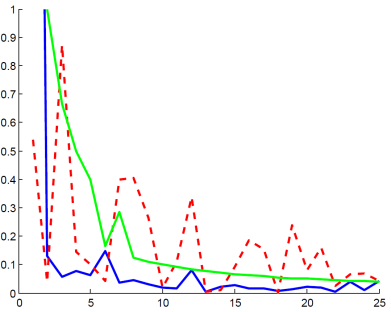

(d)

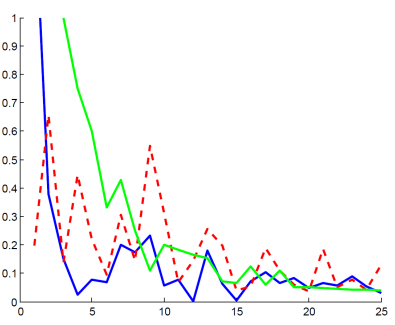

(f)
Fig. 4: Relative error err of the estimated radius for radii ranging from 1 to 25 pixels and a Gaussian PSF with (a-b) $\sigma_{P S F}=0.5$, (c-d) $\sigma_{P S F}=1$, (e-f) $\sigma_{P S F}=2$. The first column shows the result for profiles with no noise, while a $10 \%$ random Gaussian noise has been added to vessel templates in the second column (see the text for further details).

centerlines and a segmentation reconstructed from both types of information are illustrated in Fig. 5. Most vessels are correctly recovered, with accurate radii (they are slightly overestimated in the case of very small vessels, as one should expect from Sect. 4.1).

\section{CONCLUSION}

We presented a new segmentation-free method to extract scale information of vascular structures from the GVF of an image, without any additional multiscale analysis. We demonstrated that, through fast and effective one-dimensional analysis of the GVF, we are able to devise a method which is both accurate and robust to noise. The result can serve as an input for deformable model-based algorithms, to further refine the segmentation. The current bottleneck of our approach lies in the computation of the GVF which is highly timeconsuming, as any processes involving diffusion. Efforts will be put on efficient schemes to solve this variational problem. In the future, we believe that our approach will prove to be a good alternative to multiscale analysis.

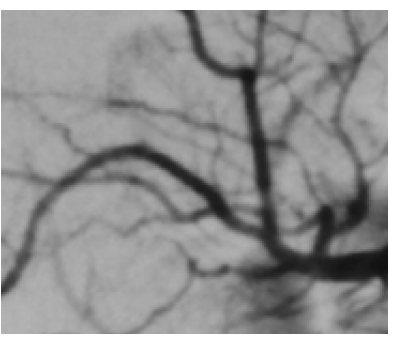

(a)

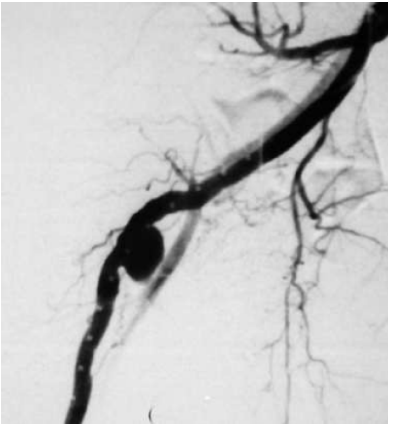

(c)

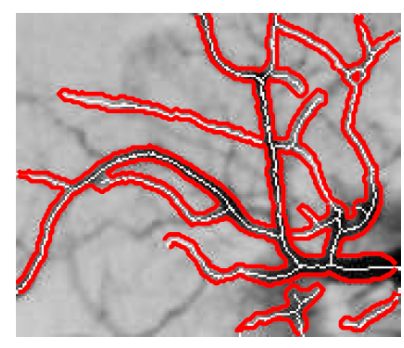

(b)

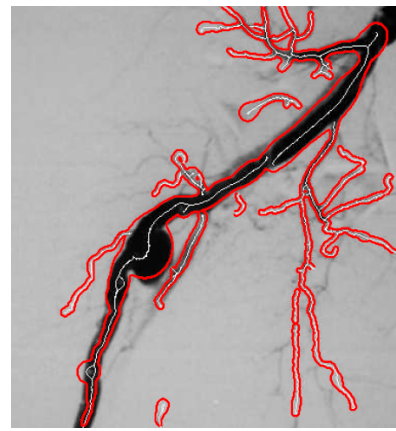

(d)
Fig. 5: Two examples of centerlines extracted from the medialness map $\mathcal{M}$ and their corresponding vessel segmentation, on 2D X-ray angiographies.

\section{REFERENCES}

[1] Xu, C. and Prince, J.L., "Snakes, Shapes, and Gradient Vector Flow," IEEE Transactions on Image Processing, vol. 7, no. 3, pp. 359-369, 1998.

[2] Xu, C. and Prince, J.L., "Global Optimality of Gradient Vector Flow," Proc. of 34th Annual Conference on Information Sciences and Systems, pp. 1-2, 2000.

[3] Xu, C. and Prince, J.L., "Generalized Gradient Vector Flow External Forces for Active Contours," Signal Processing, vol. 71, pp. 131-139, 1999.

[4] Bauer, C., Bischof, H. and Beichel, R., "Segmentation of Airways Based on Gradient Vector Flow," International Workshop on Pulmonary Image Analysis (Medical Image Computing and Computer Assisted Intervention), pp. 191-201, 2009.

[5] Engel, D. and Curio, C., "Scale-invariant Medial Features Based on Gradient Vector Flow Fields," Proc. of 19th International Conference on Pattern Recognition, pp. 1-4, 2008.

[6] Yu, Z. and Bajaj, C., "A Segmentation-Free Approach for Skeletonization of Gray-Scale Images via Anisotropic Vector Diffusion," Computer Vision and Pattern Recognition, vol. 1, pp. 415-420, 2004.

[7] Hassouna, M.S. and Farag, A.A., "On the Extraction of Curve Skeletons using Gradient Vector Flow," Proc. of 11th International Conference on Computer Vision, pp. 1-8, 2007.

[8] Boukerroui, D., "Efficient Numerical Schemes for Gradient Vector Flow," Proc. of 16th International Conference on Image Processing, pp. 4057-4060, 2009. 